\title{
Comparison of the effect of terlipressin and albumin on arterial blood volume in patients with cirrhosis and tense ascites treated by paracentesis: a randomised pilot study
}

\author{
R Moreau, T Asselah, B Condat, C de Kerguenec, F Pessione, B Bernard, T Poynard, \\ M Binn, JD Grangé, D Valla, D Lebrec
}

See end of article for authors' affiliations

Correspondence to:

Correspondence to:
Dr R Moreau, INSERM U-481, Hôpital Beaujon, 92118, Clichy, France; rmoreau@bichat.inserm.fr

Accepted for publication 9 April 2001

\begin{abstract}
Background: Patients with cirrhosis and tense ascites treated by paracentesis alone have a decrease in effective arterial blood volume after ascites removal. Although intravenous albumin is effective in preventing paracentesis induced decreased arterial blood volume, its clinical use is controversial. As paracentesis induces arteriolar vasodilation which plays a role in the development of decreased effective arterial blood volume, administration of a vasoconstrictor (terlipressin) could prevent circulatory alterations due to paracentesis.

Aims: To perform a pilot study comparing the effects of terlipressin and albumin on effective arterial blood volume in patients with cirrhosis treated by paracentesis for tense ascites.

Methods: Twenty patients with cirrhosis and tense ascites were randomly assigned to be treated by either paracentesis and terlipressin or paracentesis and albumin. Terlipressin $(3 \mathrm{mg})$ or albumin $(8 \mathrm{~g} / \mathrm{l}$ of removed ascites) were administered on the day of paracentesis. Effective arterial blood volume was assessed by measuring plasma renin concentrations at baseline and on the day of hospital discharge (4-6 days after treatment). Decreased effective arterial blood volume was defined as an increase in plasma renin concentrations on the day of hospital discharge of more than $50 \%$ of baseline values. Results: Irrespective of the treatment group, mean values for plasma renin concentrations at hospital discharge did not differ from their respective baseline values $(p=0.10)$. Baseline plasma levels of renin concentrations did not differ between the terlipressin and albumin groups $(p=0.61)$. Changes from baseline in plasma renin concentrations did not differ between groups $(p=0.39)$. Three patients in the terlipressin group and three in the albumin group developed decreased arterial blood volume.

Conclusions: This randomised pilot study suggests that terlipressin may be as effective as intravenous albumin in preventing a decrease in effective arterial blood volume in patients with cirrhosis treated by paracentesis for tense ascites.
\end{abstract}

bitl herapeutic (that is, total) paracentesis is used in patients with cirrhosis and tense ascites. ${ }^{12}$ In some studies paracentesis alone was found to induce a decrease in effective arterial blood volume (which is indirectly measured by increased activity in the renin-angiotensin system) ${ }^{3-6}$ while in other studies arterial blood volume was not altered..$^{7}$ The decrease in arterial blood volume starts after the end of paracentesis and may persist for months. ${ }^{9-11}$ Decreased effective arterial blood volume may induce inhospital complications such as impaired renal function or hyponatraemia. ${ }^{410}{ }^{11}$ Moreover, decreased arterial blood volume may be associated with a significant reduction in long term survival..$^{10}$ Although intravenous albumin administration after paracentesis has been shown to prevent the postparacentesis decrease in arterial blood volume, ${ }^{9-13}$ its clinical use is controversial ${ }^{10}{ }^{14}{ }^{15}$; for example, it is used extensively in Europe but not in North America. ${ }^{2}$ Paracentesis also induces arteriolar vasodilation which plays a major role in initiating the decrease in arterial blood volume..$^{3516}$ Therefore, administration of a vasoconstrictor may decrease paracentesis induced arteriolar vasodilation and prevent the resulting decrease in effective arterial blood volume. However, the effects of a vasoconstrictor on paracentesis induced circulatory alterations have not yet been studied. Therefore, before planning a trial including a large series of patients, it was considered important to perform a randomised pilot study comparing the effects of terlipressin (a vasopressin analogue and a potent vasoconstrictor $)^{17}$ and albumin on effective arterial blood volume in patients with cirrhosis treated by paracentesis for tense ascites.

\section{PATIENTS AND METHODS \\ Patients}

A total of 24 consecutive patients with histologically proved cirrhosis and tense ascites who were admitted to three university hospitals between June 1998 and March 2000 were enrolled in the study. The study was approved by the ethics committee (Comité Consultatif de Protection des Personnes dans la Recherche Biomédicale de Paris-Bichat Claude Bernard), and patients gave written informed consent to participate. Inclusion criteria were the presence of tense ascites requiring therapeutic paracentesis; patients less than 70 years of age; absence of arterial hypertension, a history of coronary heart disease, cardiac failure, symptomatic arteritis, respiratory or renal disease, diabetes mellitus, hepatocellular carcinoma, and hepatic encephalopathy; absence of sepsis and gastrointestinal bleeding within seven days before the study; and absence of recent use (within seven days) of diuretics, plasma expanders, or paracentesis.

Patients were randomly assigned to one of two treatment groups: 11 patients were assigned to treatment with terlipressin and 13 patients to treatment with albumin. Randomisation was performed independently at each hospital with the use of sealed envelopes containing the treatment assignments. For ethical reasons there was no placebo group in the study. ${ }^{10}$ Three patients were withdrawn from the study before paracentesis because they no longer fulfilled the inclusion criteria. One patient in the terlipressin group received saline hydration to prevent radiocontrast induced nephropathy. One patient in the albumin group had gastrointestinal 
bleeding requiring intensive medical care and another patient in the same group did not undergo paracentesis because ascites was compartmentalised. None of these three patients received the assigned treatment. A fourth patient in the albumin group received the assigned treatment but was withdrawn because he had a very high baseline plasma renin level $(2550 \mathrm{pg} / \mathrm{ml})$ for unexplained reasons. Therefore, a total of four patients were excluded from all analyses.

\section{Protocol}

Patients were studied when they were clinically stable and after having received a low sodium diet ( $34 \mathrm{mmol} / \mathrm{day}$, with no limitation on fluid intake) for at least three days. On the third day, body weight was measured and spontaneously voided urine was collected to measure sodium excretion. On the fourth day, at 0800, arterial pressure, heart rate, electrocardiogram, liver and renal tests, and blood samples for measurement of plasma concentrations of renin and aldosterone were obtained in all patients in the supine position. On the same day, at 0900, all patients underwent total paracentesis (complete removal of ascites in a single tap at a rate of $2.5 \mathrm{l} / \mathrm{h}$ ), as previously described..$^{18}$

A total dose of $3 \mathrm{mg}$ terlipressin was administered as an intravenous bolus of $1 \mathrm{mg}$ terlipressin (Glypressine; Laboratoire Ferring SAS, Gentilly, France) at the onset of paracentesis and then eight and 16 hours after the first bolus, in patients assigned to receive terlipressin. In patients assigned to receive albumin, albumin infusion (Albumine humaine 20\%; Laboratoire Français du Fractionnement et des Biotechnologies, Les Ulis, Courtabœuf, France) was started at the end of paracentesis. The dose of albumin was $8 \mathrm{~g} / \mathrm{l}$ of removed ascites. Fifty per cent of the dose was infused within two hours and the remaining 50\% was given six hours after the procedure. ${ }^{10}$ After treatment, paracentesis, diuretics, and plasma expanders were not allowed until hospital discharge. The delay between paracentesis and hospital discharge (that is, 4-6 days) was part of the protocol.

At 0800 on the day of hospital discharge, arterial pressure, heart rate, electrocardiogram, renal tests, and blood samples for measurement of plasma concentrations of renin and aldosterone were obtained. Values for plasma concentrations of renin and aldosterone were blindly determined after patients were discharged and based on blood samples obtained at baseline and at hospital discharge.

After hospital discharge, treatment of ascites was determined by each patient's attending hepatologist, and patients were followed up for survival.

\begin{tabular}{|c|c|c|}
\hline & $\begin{array}{l}\text { Terlipressin+ } \\
\text { paracentesis } \\
\text { group }(n=10)\end{array}$ & $\begin{array}{l}\text { Albumin }+ \\
\text { paracentesis } \\
\text { group }(n=10)\end{array}$ \\
\hline Age (y) & $50(2)$ & $58(2)$ \\
\hline Male & 8 & \\
\hline \multicolumn{3}{|l|}{ Aetiology of cirrhosis } \\
\hline Alcohol & 8 & 9 \\
\hline Hepatitis $C$ virus & 2 & 1 \\
\hline Serum bilirubin ( $\mu \mathrm{mol} / \mathrm{l})$ & $44(13)$ & $39(5)$ \\
\hline Serum albumin $(\mathrm{g} / \mathrm{l})$ & $28.4(2.0)$ & $29.6(2.0)$ \\
\hline Prothrombin (\%) & $58(5)$ & $49(3)$ \\
\hline Pugh's score & $9.4(0.5)$ & $9.6(0.5)$ \\
\hline \multicolumn{3}{|l|}{ Pugh's grade } \\
\hline B & 6 & 6 \\
\hline C & 4 & 4 \\
\hline Serum creatinine $(\mu \mathrm{mol} / \mathrm{l})$ & $75(9)$ & $74(4)$ \\
\hline Serum sodium (mmol/li) & 135 (1) & 134 (1) \\
\hline
\end{tabular}

Data are mean (SEM) or number of patients.

There were no significant differences between the two groups, except for age $(p=0.03)$.

\section{Statistical analysis}

Data were analysed using SAS (version 6.12) for Windows. Baseline patient characteristics (demographic and clinical features as well as laboratory results) were compared between the two groups by independent $t$ tests or the $\chi^{2}$ test as appropriate.

The primary aim of the study was to compare changes in effective arterial blood volume in patients treated by paracentesis and terlipressin with those treated by paracentesis and albumin. Variations in renin-angiotensin system activity are more sensitive and specific as markers of changes in effective arterial blood volume than variations in other variables. ${ }^{4}{ }^{10}$ Plasma concentrations of renin and aldosterone were used to assess the renin-angiotensin system. ${ }^{19}$ Two factor repeated measures ANOVA was used for analyses. The first factor analysed the nature of treatment (terlipressin or albumin) and the second factor analysed the repetition of measures (that is, at baseline and at hospital discharge). Comparisons of plasma renin concentrations were performed after logarithmic transformation. In addition, the number of patients who developed decreased arterial blood volume was compared between the terlipressin and albumin groups using the $\chi^{2}$ test. The criteria used to define decreased arterial blood volume were derived from those used in previous studies. ${ }^{10}{ }^{11}$ In the present study, decreased arterial blood volume was defined as an increase in plasma renin concentration of more than $50 \%$ of baseline values to a level greater than $50 \mathrm{pg} / \mathrm{ml}$ on the day of hospital discharge. The latter is the upper value for plasma renin concentration found in our laboratory in normal subjects receiving a low sodium diet.

The secondary aim of the study was to compare treatment induced changes in serum creatinine and serum sodium between the two groups. In addition, the number of patients who developed renal impairment or hyponatraemia after treatment were compared between the two groups. Renal impairment was defined as an increase in serum creatinine of more than $50 \%$ from baseline values, to a level greater than $133 \mu \mathrm{mol} / \mathrm{l}^{4}$ Hyponatraemia was defined as a decrease in serum sodium of more than $5 \mathrm{mmol} / \mathrm{l}$, to a level below $130 \mathrm{mmol} / \mathrm{l}^{4}$ Patients with baseline serum sodium below $130 \mathrm{mmol} / \mathrm{l}$ who had a decrease in serum sodium of at least $5 \mathrm{mmol} / \mathrm{l}$ after treatment were also included among patients in whom hyponatraemia developed. ${ }^{4}$ Moreover, the study compared treatment induced changes in body weight and arterial pressure between groups. Comparisons were performed using two factor repeated measures ANOVA or the $\chi^{2}$ test as appropriate. Finally, survival curves were compared between the two treatment groups using the log rank test. Results are expressed as mean (SEM). A two tailed $p$ value less than 0.05 was considered significant.

\section{RESULTS}

Except for age, the two treatment groups were matched for demographic and clinical features, as well as for liver tests (table 1). Age did not have any significant influence on the results. The volume of removed ascites was 6.0 (1.0) litres in the terlipressin group and $4.9(0.8)$ litres in the albumin group $(\mathrm{p}=0.42)$. The delay between paracentesis and hospital discharge was $5.5(0.2)$ days in the terlipressin group and 5.3 (0.3) days in the albumin group $(\mathrm{p}=0.57)$.

Baseline values for plasma renin concentrations did not differ from values at hospital discharge irrespective of the treatment group ( 171 (33) $v 174$ (34) pg/ml; $\mathrm{p}=0.99$ ). Individual changes in plasma renin concentrations in the two treatment groups are shown in fig 1 . Three patients had a decrease in arterial blood volume in the terlipressin group and three in the albumin group (increases in plasma renin concentration by 185 (72) and 225 (143)\%, respectively). The number of patients who had decreased arterial blood volume did not differ between the two groups $(p=0.74)$. Mean values for 

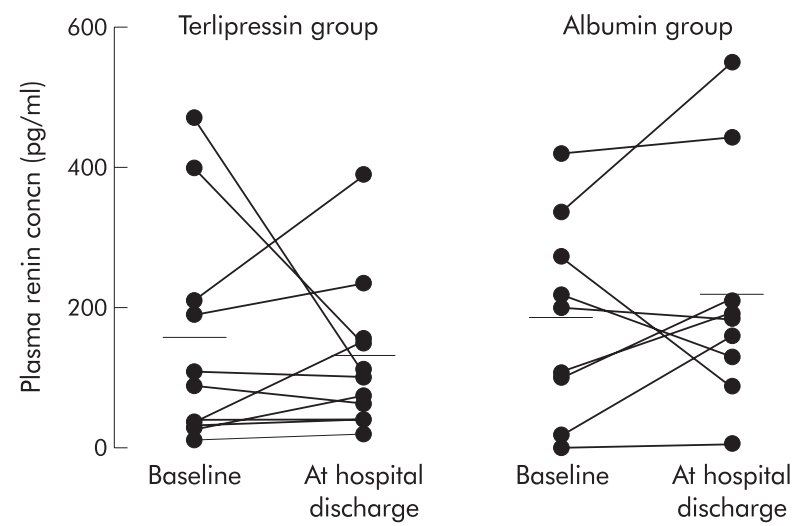

Figure 1 Individual changes in plasma renin concentrations in the terlipressin and albumin groups. Plasma renin concentrations were measured at baseline (immediately before paracentesis) and at hospital discharge (4-6 days after paracentesis).

baseline plasma renin concentrations were similar in the terlipressin and albumin groups $(\mathrm{p}=0.61)$. Changes in plasma renin concentrations (from $156(81)$ to $130(34) \mathrm{pg} / \mathrm{ml}$ in the terlipressin group and from 193 (47) to 223 (57) pg/ml in the albumin group) did not differ between groups (table 2 ).

Baseline plasma aldosterone concentrations did not differ from values at hospital discharge (1213 (237) v 1377 (197) $\mathrm{pg} / \mathrm{ml} ; \mathrm{p}=0.13$ ) in either treatment group. Baseline plasma aldosterone concentrations did not differ between the terlipressin and albumin groups $(\mathrm{p}=0.42)$. Changes in plasma aldosterone concentrations (from $1017(278)$ to $1336(280)$ $\mathrm{pg} / \mathrm{ml}$ in the terlipressin group and from 1149 (324) to 1421 (292) $\mathrm{pg} / \mathrm{ml}$ in the albumin group) did not differ between groups (table 2 ). In patients with a decrease in effective arterial blood volume, plasma aldosterone concentrations increased by $145(101) \%$ and $263(117) \%$ in the terlipressin and albumin groups, respectively.

Plasma concentrations of renin and aldosterone measured on the day after paracentesis were available in eight patients in the terlipressin group and in seven in the albumin group. Plasma renin concentrations measured at baseline and on the day after paracentesis were $166(62)$ and 55 (22) pg/ml in the terlipressin group, and 192 (59) and $166(70) \mathrm{pg} / \mathrm{ml}$ in the albumin group. Plasma aldosterone concentrations measured at baseline and on the day after paracentesis were 928 (299) and 495 (252) pg/ml in the terlipressin group, and 1151 (361) and $500(70) \mathrm{pg} / \mathrm{ml}$ in the albumin group.

Baseline serum creatinine levels did not differ from values at hospital discharge (74 (5) $v 72(5) \mu \mathrm{mol} / \mathrm{l} ; \mathrm{p}=0.29)$ in the two treatment groups. Baseline serum creatinine did not differ between the terlipressin and albumin groups $(p=0.93)$.
Changes in serum creatinine (from 75 (9) to 72 (8) $\mu \mathrm{mol} / \mathrm{l}$ in the terlipressin group and from 74 (4) to 71 (6) $\mu \mathrm{mol} / \mathrm{l}$ in the albumin group) did not differ between groups (table 2 ). No patient developed renal impairment after treatment in the terlipressin or albumin group.

Serum sodium was lower at hospital discharge than at baseline (132 (1) $v 134$ (1) $\mathrm{mmol} / \mathrm{l} ; \mathrm{p}<0.001)$ for both treatment groups. Baseline serum sodium was similar in the terlipressin and albumin groups $(\mathrm{p}=0.56)$. Changes in serum sodium (from 134 (1) to 133 (1) $\mathrm{mmol} / \mathrm{l}$ in the terlipressin group and from 134 (1) to 132 (1) $\mathrm{mmol} / \mathrm{l}$ in the albumin group) did not differ between groups (table 2 ). The number of patients who developed hyponatraemia after treatment was not different between the two groups (one patient in the terlipressin group and one in the albumin group; $\mathrm{p}=0.46$ ).

In both treatment groups, body weight was lower at hospital discharge than at baseline (71 (3) $v 75$ (3) kg; p<0.001). Baseline body weight did not differ between the terlipressin and albumin groups $(p=0.40)$. Changes in body weight (from 77 (4) to 73 (3) kg in the terlipressin group and from 72 (5) to 69 (4) kg in the albumin group) did not differ between groups (table 2).

Baseline values for mean arterial pressure did not differ from values at hospital discharge (80 (2) $v 78$ (2) mm Hg; $p=0.55$ ) for either treatment group. Baseline arterial pressure did not differ between the terlipressin and albumin groups $(p=0.77)$. Changes in arterial pressure (from 79 (3) to 78 (3) $\mathrm{mm} \mathrm{Hg}$ in the terlipressin group and from 81 (4) to 79 (3) mm $\mathrm{Hg}$ in the albumin group) did not differ between groups (table 2).

Baseline values for urinary sodium excretion did not differ from values at hospital discharge (9 (2) $v 7$ (1) mmol/day; $\mathrm{p}=0.13$ ) for either treatment group. Baseline urinary sodium excretion did not differ between the terlipressin and albumin groups $(p=0.42)$. Changes in urinary sodium excretion (from 11 (4) to 7 (3) mmol/day in the terlipressin group and from 7 (3) to 7 (2) $\mathrm{mmol} / \mathrm{day}$ in the albumin group) did not differ between groups $(\mathrm{p}=0.25)$.

In the two groups, there were no differences between baseline values and values at hospital discharge for heart rate, serum potassium, or serum bicarbonate. Baseline values of these variables did not differ significantly between the two groups (data not shown).

Inhospital complications did not occur in either group. In the terlipressin group, abdominal cramps, diarrhoea, arterial hypertension, and chest pain were not observed. There were no differences between pretreatment and post-treatment electrocardiograms (data not shown).

At three months the survival rate did not differ between the two groups $(86 \%$ in the terlipressin group and $89 \%$ in the albumin group; $\mathrm{p}=0.86$ ).

\begin{tabular}{|c|c|c|c|}
\hline & $\begin{array}{l}\text { Terlipressin+ } \\
\text { paracentesis } \\
\text { group }(n=10)\end{array}$ & $\begin{array}{l}\text { Albumin }+ \\
\text { paracentesis } \\
\text { group }(n=10)\end{array}$ & $\mathrm{p}$ Value \\
\hline \multicolumn{4}{|l|}{ Changes in* } \\
\hline \multicolumn{4}{|l|}{ Plasma concentrations (pg/ml) } \\
\hline Renin & $-27(51)$ & $30(37) \dagger$ & 0.39 \\
\hline Aldosterone & $319(246)$ & $272(281) \dagger$ & 0.90 \\
\hline Serum creatinine $(\mu \mathrm{mol} / \mathrm{l})$ & $-2.4(2.9)$ & $-2.3(3.2)$ & 0.98 \\
\hline Serum sodium (mmol/l) & $-1.6(0.8)$ & $-1.9(0.6)$ & 0.78 \\
\hline Body weight $(\mathrm{kg})$ & $-3.4(0.9)$ & $-3.3(1.0)$ & 0.91 \\
\hline Mean arterial pressure $(\mathrm{mm} \mathrm{Hg})$ & $-1.3(3.2)$ & $-1.2(2.8)$ & 0.98 \\
\hline \multicolumn{4}{|c|}{$\begin{array}{l}\text { *Changes were the difference between baseline values (before treatment) and values } \\
\text { measured at hospital discharge ( } 4-6 \text { days after treatment). Negative changes indicate a } \\
\text { decrease. } \\
\text { Values are mean (SEM). } \\
\text { †Measured in nine patients. }\end{array}$} \\
\hline
\end{tabular}




\section{DISCUSSION}

In this pilot study, patients with cirrhosis and tense ascites were randomly assigned to be treated with either paracentesis plus terlipressin or paracentesis plus albumin. Albumin was used because to date it is the best treatment for preventing inhospital complications of paracentesis. ${ }^{10}$ Under baseline conditions, the groups were similar for liver tests, plasma levels of renin and aldosterone, arterial pressure, serum creatinine, and serum sodium. Patients were in a poor condition in both groups.

The main aim of the study was to compare changes in effective arterial blood volume assessed by measuring variations in renin-angiotensin system activity-that is, changes in plasma renin concentrations. ${ }^{10}{ }^{19}$ Plasma renin and aldosterone concentrations were measured immediately before the onset of paracentesis and at hospital discharge (4-6 days after paracentesis). In addition, plasma levels of renin and aldosterone were measured on the day after paracentesis in some patients in both groups.

As expected, ${ }^{90}$ in the albumin group plasma renin and aldosterone concentrations were lower on the day after paracentesis than under baseline conditions. Similarly, in the terlipressin group, plasma renin and aldosterone concentrations were lower on the day after paracentesis than at baseline.

Patients with tense ascites treated by paracentesis alone have a significant decrease in effective arterial blood volume, as indicated by marked increases in plasma renin activity and plasma aldosterone levels within one week after ascites removal. ${ }^{49}{ }^{10}$ In the present study, a decrease in arterial blood volume was present on the day of hospital discharge in only three patients in each treatment group. Moreover, mean values for plasma concentrations of renin and aldosterone did not change significantly in the terlipressin and albumin groups. Thus paracentesis induced decreased effective arterial blood volume was effectively prevented in most patients in the terlipressin and albumin groups.

Paracentesis induced arteriolar vasodilation precedes and plays a major role in the development of decreased effective arterial blood volume. ${ }^{31116}$ Terlipressin is a potent vasoconstrictor, ${ }^{17}$ and as terlipressin administration started immediately before ascites removal, prevention of paracentesis induced vasodilation may explain the lack of change in effective arterial blood volume in patients who received terlipressin.

Another aim of the present study was to compare changes in clinically significant variables between the two groups. In both groups, the mean value for serum creatinine at hospital discharge did not differ significantly from baseline values and no individual patient developed renal impairment during the study. In addition, although each treatment group showed a slight but significant decrease in natraemia, changes in serum sodium were similar in the two groups. Moreover, hyponatraemia occurred in only one patient in each group. The results also show that body weight was significantly lower on the day of hospital discharge than on the day before paracentesis in the terlipressin and albumin groups. Decreases in body weight (that are obviously a result of ascites removal) were similar in both treatment groups. Finally, arterial pressure at hospital discharge did not differ significantly from baseline values in the terlipressin and albumin groups. These results, together with those obtained for plasma renin levels (see above), indicate that the overall response to treatment was similar in the terlipressin and albumin groups. However, because of the small sample size of the study groups, a type II error cannot be excluded.

In this study, terlipressin was well tolerated in all patients, as in other previous studies. ${ }^{17}{ }^{21}$ In contrast, albumin is a blood product that may have inherent risks, such as transmission of diseases. The cost of terlipressin was slightly less than that of intravenous albumin (994 $v 1100$ Euros, respectively). It should be emphasised that the cost of terlipressin was fixed while that of albumin increased with the volume of ascites removed and thus albumin may cost less or, more frequently, more than terlipressin.

In conclusion, the results of this randomised pilot study suggest that the use of terlipressin may be a novel therapy in the prevention of the paracentesis induced decrease in arterial blood volume. Therefore, the effects of terlipressin on arterial blood volume should be compared with those of intravenous albumin in a randomised study involving a large series of patients with cirrhosis and tense ascites treated by paracentesis.

\section{ACKNOWLEDGMENTS}

The authors thank Dr Isabelle Colle, Dr Philippe Langlet, and Alain Truskolaski. Terlipressin was a kind gift from Ferring SAS (Gentilly, France). The study was supported by the Assistance PubliqueHôpitaux de Paris.

\section{Authors' affiliations}

R Moreau, T Asselah, B Condat, C de Kerguenec, D Valla,

D Lebrec, Laboratoire d'Hémodynamique Splanchnique et de Biologie Vasculaire, INSERM U-481 and Service d'Hépatologie, Hôpital Beaujon, Clichy, France

F Pessione, UTAMA, Hôpital Beaujon, Clichy, France

B Bernard, T Poynard, Service d'Hépato-Gastroentérologie, Groupe Hospitalier Pitié-Salpêtrière, Paris, France

M Binn, JD Grangé, Service d'Hépato-Gastroentérologie, Hôpital

Tenon, Paris, France

\section{REFERENCES}

1 Quintero E, Ginés P, Arroyo V, et al. Paracentesis versus diuretics in the treatment of cirrhotics with tense ascites. Lancet 1985;1:611-12.

2 Runyon BA. Management of adult patients with ascites caused by cirrhosis. Hepatology 1998;27:264-72.

3 Simon DM, McCain JR, Bonkovsky HL, et al. Effects of therapeutic paracentesis on systemic and hepatic hemodynamics and on renal and hormonal function. Hepatology 1987;7:423-9.

4 Ginès $\mathbf{P}$, Tito LI, Arroyo V, et al. Randomized comparative study of therapeutic paracentesis with and without intravenous albumin in cirrhosis. Gastroenterology 1988;94:1493-502.

5 Panos MZ, Moore K, Vlavianos P, et al. Single, total paracentesis for tense ascites: sequential hemodynamic changes and right atrial size. Hepatology 1990;11:662-7

6 Wang SS, Lu CW, Chao Y, et al. Total paracentesis in non-alcoholic cirrhotics with massive ascites: mid-term effects on systemic and hepatic haemodynamics and renal function. J Gastroenterol Hepatol 1994;9:592-6.

7 Kao HW, Koc NE, Savage E, et al. The effect of large volume paracentesis on plasma volume. A cause of hypovolemia? Hepatology 1985;5:403-7.

8 Pinto PC, Amerian J, Reynolds TB. Large-volume paracentesis in nonedematous patients with tense ascites: its effect on intravascular volume. Hepatology 1988;8:207-10.

9 Luca A, Garcia-Pagan JC, Bosch J, et al. Beneficial effects of intravenous albumin infusion on the hemodynamic and humoral changes after total paracentesis. Hepatology 1995;22:753-8.

10 Ginès A, Fernandez-Esparrach G, Monescillo $A$, et al. Randomized trial comparing albumin, Dextran 70, and polygeline in cirrhotic patients with ascites treated by paracentesis. Gastroenterology 1996:111:1002-10.

11 Ruiz-Del-Arbol L, Monescillo A, Jiménez W, et al. Paracentesis-induced circulatory dysfunction: mechanism and effect on hepatic hemodynamics in cirrhosis. Gastroenterology 1997;113:579-86.

12 Salerno F, Badalamenti S, Incerti P, et al. Repeated paracentesis and i.v. albumin infusion to treat 'tense' ascites in cirrhotic patients. A safe alternative therapy. J Hepatol 1987;5:102-8.

13 Pozzi M, Osculati G, Boari G, et al. Time course of circulatory and humoral effects of rapid total paracentesis in cirrhotic patients with tense, refractory ascites. Gastroenterology 1994;106:709-19.

14 Runyon BA. Albumin infusion for spontaneous bacterial peritonitis. Lancet 1999;354:1838-9.

15 Cochrane Injuries Group Albumin Reviewers. Human albumin administration in critically patients: systematic review of randomised controlled trials. BM 1998;317:235-40.

16 Vila MC, Sola R, Molina L, et al. Hemodynamic changes in patients developing effective hypovolemia after total paracentesis. J Hepatol 1998;28:639-45.

17 Gadano A, Moreau R, Vachiery F, et al. Natriuretic response to the combination of atrial natriuretic peptide and terlipressin in patients with cirrhosis and refractory ascites. J Hepatol 1997;26:1229-34. 
18 Lebrec D, Giuily N, Hadengue A, et al. Transjugular intrahepatic portosystemic shunts: comparison with paracentesis in patients with cirrhosis and refractory ascites. A randomized trial. J Hepatol 1996;25:135-44.

19 Asbert M, Jiménez W, Gaya J, et al. Assessment of the renin-angiotensin system in cirrhotic patients. Comparison between plasma renin activity and direct measurement of immunoreactive renin. J Hepatol 1992;15:179-83.
20 Garcia-Compean D, Villareal JZ, Cuevas HB, et al. Total therapeutic paracentesis (TTP) with and without intravenous albumin in the treatment of cirrhotic tense ascites: a randomized controlled trial. Liver 1993;13:233-8.

21 Hadengue A, Gadano A, Moreau R, et al. Beneficial effects of the two-day administration of terlipressin in patients with cirrhosis and hepatorenal syndrome. J Hepatol 1998;29:565-70.

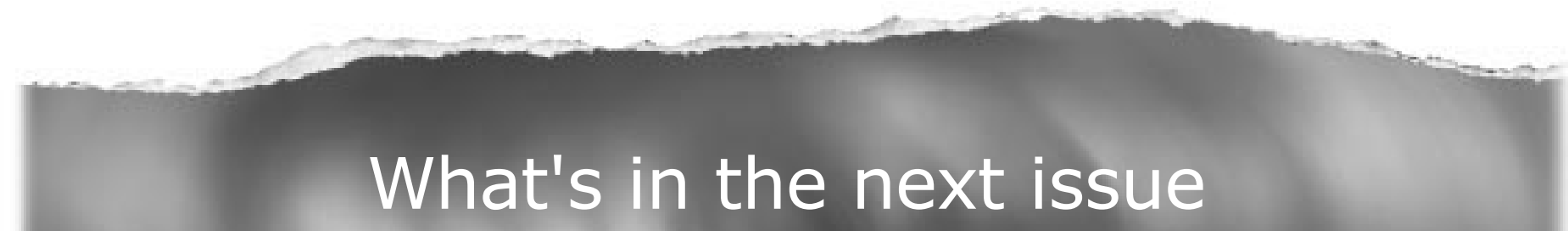

\section{Future content}

See which articles have just been accepted for publication and preview the table of contents for the next issue a month before it is published

www.gutjnl.com 